\section{Seven years of L-dopa therapy}

by Miranda Robertson

Nominally, the symposium held on June 28 by the Parkinson's Disease Society was to mark the fact that it is now 150 years since the death of James Parkinson. What was most clearly reflected in its content, however, was that it is now seven years since the advent of L-dopa therapy, which seemed at one time to promise freedom from parkinsonism but has turned out merely to suspend sentence. This has left pharmacologists to try and find out what to do next and clinicians with the problem of what best to do in the meantime; and that, apart from an historical excursion by J. D. Parkes (Institute of Psychiatry, London), was what the symposium was about.

There has been no radical change in the therapeutic approach to parkinsonism since Charcot first used anticholinergic drugs a century ago. The aim is still to redress the balance between cholinergic and dopaminergic agonists in the basal ganglia, but with the emphasis on supplying the missing dopamine rather than blocking acetylcholine reception. K. Fuxe (Karolinksa Institute, Stockholm) favoured ergot derivatives and in particular 2-bromoalpha-ergocryptine (CB154) as future alternatives to dopamine derived from $L$-dopa. The advantages of these dopaminergic agonists over L-dopa would be that they act directly on the dopamine receptor without having to be converted to the active form by enzymes which may be deficient as a result of the same degenerative process that produces the dopamine deficiency. Fuxe, who has tried CB154 on rats with nigro-striatal lesions, is optimistic about the compound whose effects, though weaker than those of L-dopa, are more prolonged. His hope, however, that CB154 might also be free of some of its side effects is not substantiated by preliminary clinical trials, reported by D. Calne (Hammersmith Hospital, London). Functional improvement was assessed blind at between $9 \%$ and $18 \%$ on average for 20 patients on the drug, the degree of improvement being positively correlated with the severity of the disability. But adverse reactions seemed to parallel those seen with L-dopa therapy.

Amplification of post-synaptic effects through the adenyl cyclase system, which is also under investigation at the Karolinska Institute, may conceivably help with the separation of therapeutic from side effects, although as C. D. Marsden (King's College Hospital, London) pointed out, it is not clear how different the receptors mediating the one are from those mediating the other. In Fuxe's rat model, however, theophylline (which inhibits phosphodiesterase and thus increases the concentration of cyclic AMP) does seem to increase activity in the denervated striatal neurones, and he believes that it may be possible to use the drug to potentiate the effects of CB154.

All this, however, assumes a functional post-synaptic receptor mechanism, and as M. Sandler (Queen Charlotte's Hospital, London) pointed out, receptor pathology is by no means ruled out. Sandler, who has long held that the dopamine replacement theory of L-dopa therapy is an oversimplification, devoted much of his talk to hauling skeletons out of cupboards. One of them is ignorance of the exact cause of Parkinson's disease, and of the contribution of deficiencies in chemicals other than dopamine to the syndrome. The other is the failure to explain either how L-dopa works or why it stops working. Neither the disease nor dopa metabolism, in short, is understood.

What seems to be missing is a really good animal model for parkinsonism. Sandler stressed that drugs do not always have the same effect on lesioned rats as they do on parkinsonian humans. This is hardly surprising if only because the parkinsonian lesion is most unlikely to be as simple as the destruction of the nigro-striatal pathway, besides which, autopsy brains of parkinsonian patients show evidence (quoted by M. Yahr, Mount Sinai School of Medicine) of continuing degeneration under L-dopa therapy; whereas the surgical lesion does not evolve.

There is also the question of the effect of prolonged administration of drugs. Clinicians repeatedly brought up the need at the very least for studies on the effects of anti-parkinsonian agents over long periods of time. Progressive disease may explain why parkinsonism in due course overcomes L-dopa therapy; but what is the explanation of the simultaneous increase in the dyskinetic side effects of the drug?

Some recent work of $\mathbf{H}$. Klawans (University of Chicago) suggests that the answer may lie in the post-synaptic dopamine receptors. $\mathrm{He}$ used amphetamine to stimulate the dopamine receptors of guinea pigs to the point at which the drug produced stereotyped movements, and found that after regular treatment over a period of time, the movements could be elicited not only by progressively lower doses of amphetamine but also by the dopaminergic agonist apomorphine.

The meeting ended with a review lecture by A. Carlson (University of Goteborg), whose efforts in establishing dopamine as a neurotransmitter in the late 1950 s helped to make possible the introduction of dopaminergic antagonists in the treatment of parkinsonism.

\section{An amoeba becomes a flagellate}

\author{
from $F . E . G . C o x$
}

Parasitology Correspondent

PARASITOlogists are tidy people and like to know how parasites should be classified. The understanding of the correct taxonomic status of a parasite is more than the provision of a handy slot in which to file information; it is the basis of an understanding of the biology of the organism and a point to which related organisms can be referred. From such a reference point stems a knowledge of basic biochemistry, physiology and epidemiology and on these studies the control of the parasite can be based. Parasites, particularly protozoa, are often very specialised and it is not always easy to see their basic structures with the light microscope. During the past decade, however, the electron microscope has been used to solve several taxonomic problems. Partially as a result of such studies the piroplasms and Toxoplasma have taken their rightful position within the sporozoan class Coccidia, and the number of discrete taxonomic groups containing a few parasites of uncertain taxonomic position has been diminished.

One of the outstanding taxonomic problems is concerned with an amoeba, Dientamoeba fragilis, which lives in the gut of man. It has long been recognised that this binucleate amoeba could be a flagellate but absolute proof has been lacking. Two independent papers in the Journal of Protozoology have now supplied this proof. Honigberg and his colleagues (Camp, Mattern and Honigberg, J. Protozool., 21, 69; 1974) now report that the fine structure of Dientamoeba places it among the trichomonad flagellates and this conclusion is also reached by Dwyer (ibid., 139) using immunoelectrophoretic methods to analyse the antigens of Trichomonas, Dientamoeba, Histomonas and Entamoeba. This latter work is a culmination of Dwyer's earlier immunological studies on these parasites (ibid., 19, 316 and $326 ; 1972)$. All the evidence now points to common structures and common antigens linking Dientamoeba with the flagellates Trichomonas and Histomonas and there is a clear evolutionary sequence in which the number of flagella are reduced in Histomonas and disappear altogether in Dientamoeba. Honigberg suggests a revised classification of the Order Trichomonadida in which Dientamoeba is placed in the same family as Histomonas and this is likely to be generally accepted by protozoologists and parasitologists.

The implications of these studies lie in an understanding of the mode of transmission of parasitic members of the order Trichomonadida, Protective 